\title{
Ultra-broadband silicon photonics devices based on subwavelength metamaterials -INVITED
}

\author{
A. V. Velasco ${ }^{1, *}$, D. González-Andrade ${ }^{1}$, A. Herrero-Bermello ${ }^{2}$, J. M. Luque-González ${ }^{3}$, R. Halir ${ }^{3}$, J. G. Wangüemert- \\ Pérez $^{3}$, A. Ortega-Moñux ${ }^{3}$, A. Dias ${ }^{2}$, I. Molina-Fernández ${ }^{3}$, and P. Cheben ${ }^{4}$ \\ ${ }^{1}$ Spanish National Research Council (CSIC), Institute of Optics, 28006 Madrid, Spain \\ ${ }^{2}$ Alcyon Photonics S.L, 28006 Madrid, Spain \\ ${ }^{3}$ Universidad de Málaga, ETSI Telecomunicación, Departamento de Ingeniería de Comunicaciones 29071 Málaga, Spain \\ ${ }^{4}$ National Research Council of Canada, Ottawa, Ontario, K1A 0R6, Canada
}

\begin{abstract}
Subwavelength structured waveguides provide tailorable optical properties that can be leveraged to overcome bandwidth limitations in a wide range of photonic devices. In this invited talk, we present an overview of recent developments on subwavelength engineered building blocks, including phase shifters, mode multiplexers, polarization beam splitters and zero-birefringence waveguides.
\end{abstract}

Subwavelength grating (SWG) metamaterials are becoming established as a fundamental tool for the development of a new generation of silicon photonics building blocks with engineered optical properties $[1,2]$. In this work, we present the fundamentals of this technology and its application to the design of several high-performance photonic devices. Firstly, we report on an ultra-broadband phase shifter [3], and on a mode converter and multiplexer/demultiplexer based on a subwavelength engineered multimode interference coupler [4]. Then, we introduce tilted SWG metamaterials, which provide an additional degree of freedom in the design by enabling to tailor the anisotropic response of the optical medium [5]. Finally, we present the application of tilted SWGs to the design of a polarization splitter $[6,7]$ and a zero-birefringence waveguide [8].

\section{Acknowledgments}

We acknowledge funding through Ministerio de Economía y Competitividad (TEC2016-80718-R, CDTI SNEO-20181232, RTI2018-097957-B-C33), Ministerio de Educación, Cultura y Deporte (FPU16/06762,), Proyecto $\mathrm{I}+\mathrm{D}+\mathrm{i}$ en el marco del Programa Operativo FEDER Andalucía 2014-2020 (UMA18-FEDERJA-219), Universidad de Málaga, Alcyon Photonics (8.06/5.59.5045), the Community of Madrid - FEDER funds (S2018/NMT-4326), and the Horizon 2020 research and innovation program under the Marie SklodowskaCurie grant No. 734331.

\section{References}

1. P. Cheben et al., "Subwavelength integrated photonics," Nature 560 (2018).

2. R. Halir et al., "Subwavelength-grating metamaterial structures for silicon photonic devices," Proc. IEEE 106 (2018).

3. D. González-Andrade et al., "Ultra-broadband nanophotonic phase shifter based on subwavelength metamaterial waveguides," Photon. Res. 8 (2020).

4. D. González-Andrade et al., "Experimental demonstration of a broadband mode converter and multiplexer based on subwavelength grating waveguides," Opt. Laser Technol. 129 (2020).

5. J. M. Luque-González et al., "Tilted subwavelength gratings: controlling anisotropy in metamaterial nanophotonic waveguides," Opt. Lett. 43 (2018).

6. A. Herrero-Bermello et al., "Design of a broadband polarization splitter based on anisotropy-engineered tilted subwavelength gratings," IEEE Photonics J. 11 (2019).

7. A. Herrero-Bermello et al., "Experimental demonstration of metamaterial anisotropy engineering for broadband on-chip polarization beam splitting," Opt. Express 28 (2020).

8. A. Herrero-Bermello et al., "Zero-birefringence silicon waveguides based on tilted subwavelength metamaterials," IEEE Photonics J. 11 (2019).

* Corresponding author: a.villafranca@csic.es 